\title{
CHOLESTEROL DOES NOT IMPROVE CRYOPROTECTIVE EFFICIENCY OF SOYBEAN LECITHIN-BASED EXTENDERS FOR BULL SEMEN
}

\section{N.V. SHISHOVA ${ }^{1}$, N.A. KOMBAROVA ${ }^{2}$, Yu.S. TARAKHOVSKY ${ }^{3}$, G.A. DAVYDOVA ${ }^{3}$, L.V. ZALOMOVA', O.Yu. SERAYA ${ }^{3}$, A.I. ABILOV ${ }^{4}$}

\begin{abstract}
${ }^{1}$ Institute of Cell Biophysics RAS, Federal Agency of Scientific Organizations, 3, ul. Institutskaya, Pushchino, Moscow Province, Russia 142290;

${ }^{2}$ Head Center for Reproduction of Farm Animals, 3, ul. Tsentralnaya, pos. Bykovo, Podolsk Region, Moscow Province, 142143 Russia, e-mail komnina@list.ru (corresponding author);

2Institute of Theoretical and Experimental Biophysics RAS, Federal Agency of Scientific Organizations, 3, ul. Institutskaya, Pushchino, Moscow Province, Russia 142290;

${ }^{4}$ L.K. Ernst All-Russian Research Institute of Animal Husbandry, Federal Agency of Scientific Organizations, 60, pos. Dubrovitsy, Podolsk Region, Moscow Province, 142132 Russia, e-mail: cryopreservation@list.ru (corresponding author) The authors declare no conflict of interests

Received December 30, 2016
\end{abstract}

\section{Abstract}

Design of plant phospholipid based extenders free of animal-derived components is urgent in farm animal semen cryopreservation. However, a complex of plant lipids, unlike the yolk complex, lacks cholesterol which plays an important role in cryoresistance of spermatozoa. Enriching spermatozoa membranes with cholesterol improves their cryotolerance (E. Mocé et al., 2010; E. Mocé et al., 2014; M.H. Fayyaz et al., 2016). We first estimated the impact of different cholesterol concentrations $(0,8,18,33$ and $50 \%)$ in soybean phospholipid mixtures both on morphology of obtained liposomes or lipid particles, and on their cryoprotective property as judged by the effect on motility when added to the extender at bull sperm cryopreservation. Liposome suspensions were prepared from commercial lecithin LeciPRO 90 («Unite-chem Co., Ltd», China) and purified cholesterol form lanolin («Sigma-Aldrich Co.», USA) by sonication at an ultrasound disintegrator UZDN-2T (NPP «Academpribor», Russia; $22 \mathrm{kHz}, 60 \mathrm{~W} / \mathrm{cm}^{2}, 5 \mathrm{~min}$ ). It was found that cholesterol did not notably influence the obtained liposomes at low concentration (8\%). At 18 and $33 \%$ cholesterol in the lipid mixture the size of the liposomes increased and averaged $66 \pm 6 \mathrm{~nm}$ and $62 \pm 11 \mathrm{~nm}$, respectively. Fifty percent cholesterol changed the lipid suspension completely resulting in complex structures formed by phospholipid-cholesterol lamellae and crystals of cholesterol monohydrate. Eight percent cholesterol did not increase a cryorpotective efficiency of lecithin, while 18-50\% cholesterol decreased its cryoprotective capacity. An increase in cholesterol concentration in the lipid mixture up to $50 \%$ ( $\approx 66 \mathrm{~mol} \%)$ led to somewhat higher spermatozoa motility compared to a minimum score observed at freezing with $33 \%$ cholesterol. The most probable cause of the negative impact of cholesterol on the cryoprotective effectiveness of lecithin suspension is impairment in liposome interaction with a sperm plasma membrane caused by condensation of phospholipid packing in a lipid bilayer.

Keywords: cattle, cryopreservation, sperm, cholesterol, phospholipids, liposomes

Cryopreserved sperm of elite producers is widely involved in farm animal reproduction and breeding. In Russia, media containing egg yolk which has high cryoprotective effect is traditionally used in bull semen freezing. In this, the complicating factors are volatility of the yolk, the high probability of contamination with dangerous pathogens, the rapid loss of protective properties during storage, the complexity of sterilization, etc. That is why much attention is recently being paid to the cryoprotective media without egg yolk or other components of animal origin [1].

It is known that the cryoprotective function of yolk is due to the presence of phospholipids of the light lipoprotein fraction [2, 3]. Plant-derived phospholipids, for example soy lecithin, could replace this component in cryoprotective media [4, 5]. However, in addition to phospholipids, the egg yolk contains a number of other substances with protective effect, in particular, cholesterol, 
completely absent in plant lipids.

Cholesterol is an important component of cytoplasmic membranes and has a great influence on cell resistance to cooling and freezing. In mammals [68], birds [9] and fish [10] the spermatozoa with high content of cholesterol and lipids in the membranes are more resistant to cold shock than those with low cholesterol. Later, cyclic oligosaccharide cyclodextrin was used for artificial enrichment of cell membranes with cholesterol [11]. Numerous experiments confirm that the enrichment of cell membranes with cholesterol enhances the tolerance of bull spermatozoa to cooling and freezing [12-15]. Consequently, it can be assumed that the inclusion of cholesterol in the composition of yolk-free media based on plant lecithins can increase cryoprotective efficiency.

Soy lecithin is introduced into cryoprotective media as liposomes, the structure and size of which depend on the method of preparation and the composition of the raw materials. Since cholesterol has a strong effect on the structure and characteristics of the lipid bilayer, it will significantly change both the properties of liposomes, and their interaction with cytoplasmic membranes [1618]. The effect of cholesterol on the bilayer membrane, in turn, depends on the phospholipid composition of the liposomes. Therefore, to obtain reliable data on the cryoprotective role of cholesterol-contacting liposomes of plant phospholipids, it is necessary not only to study their effect on spermatozoa, but also to track changes in the liposomes themselves.

The available data on how cholesterol affects the cryoprotective functions of complex liposomes are poor though this information is of special importance when improving yolk-free diluents. For the first time, we simultaneously evaluated the effect of cholesterol in different concentrations $(0,8,18,33$ and $50 \%$ ) on the physicochemical properties of liposomes and lipid particles of soybean phospholipids, as well as on the protective effect of the resulting lipid suspensions in the cryopreservation of bull semen. It was revealed that cholesterol did not increase, and in the concentration of 18-50\% even reduced the cryoprotective efficiency of lecithin.

The aim of the study was to study the dimensional characteristics and cryoprotective properties of the suspensions resulted from a mixture of soybean phospholipids and different concentrations of cholesterol.

Technique. LeciPRO 90 (Unitechem Co., Ltd, China) commercial lecithin and purified cholesterol derived from lanolin (Sigma-Aldrich Co., USA) were used. Cholesterol fractions were $0 ; 8 ; 18 ; 33$ and $50 \%$. The lipids in this ratio were dissolved in chemically pure chloroform. The chloroform was then removed with a rotary evaporator for 4-5 hours until a thin film of phospholipids was formed on the inner wall of the glass flask, after which a sucrose solution (242 $\mathrm{mM}$ sucrose, $10 \mathrm{mM}$ HEPES) was added up to $1 \mathrm{ml}$ of water per 15 $\mu \mathrm{g}$ of lecithin. The lipids were dispersed on a laboratory shaker (type 358S, ELPIN PLUS s.c., Poland) for 2 hours at room temperature. The resulting suspension, to prevent oxidation of lecithin, was purged with nitrogen, then cooled in an ice bath to $4{ }^{\circ} \mathrm{C}$ and treated for $5 \mathrm{~min}$ on an ultrasonic disintegrator UZDN-2T (NPP Akadempribor, Russia $22 \mathrm{kHz}, 60 / \mathrm{cm}^{2}$; an immersed probe of $2 \mathrm{~mm}$ in diameter).

The average hydrodynamic diameter of the liposomes or lipid particles in the suspension and their size distribution were evaluated using the dynamic light scattering method of the N5 Submicron Particle Size Analyzer (Beckman Coulter, Inc., USA) 24 hours after the suspension was prepared.

The particles were examined under an Axio Scope FL microscope (Carl Zeiss, Germany) at $\times 400$ magnification with dark field contrast.

For cryopreservation of bovine semen and evaluation of the protective 
efficacy of lecithin we used rejected ejaculates (OAO Head Center for the Reproduction of Farm Animals), in which the proportion of spermatozoa with rectilinear motility was at least $70 \%$. Lactose-glycerol diluent (242 mM lactose, $10 \mathrm{mM}$ HEPES, $55 \mathrm{mM}$ glycerol) was a cryoprotectant. Analyzed phospholipid or phospholipid-cholesterol suspensions were added to a final lecithin concentration of $3 \mathrm{mg} / \mathrm{ml}$. Egg yolk (20\%) was added to the control samples instead of the suspension. The semen was diluted with the cryoprotective medium in two stages (depending on the amount in the ejaculate) and, after 4 hours of equilibration at $4{ }^{\circ} \mathrm{C}$, frozen in open granules on plates of solid carbon dioxide (dry ice) by the standard procedure [19]. The granules were thawed in a water bath at $39-40{ }^{\circ} \mathrm{C}$ in a special solution containing $130 \mathrm{mM} \mathrm{NaCl}, 4 \mathrm{mM} \mathrm{KCl}, 1 \mathrm{mM} \mathrm{CaCl}$, $0.5 \mathrm{mM} \mathrm{MgCl}_{2}, 14 \mathrm{mM}$ fructose, $10 \mathrm{mM}$ HEPES (Merck KGaA, Germany), and $0.1 \%$ bovine serum albumin (OOO NPP PanEco, Russia).

The cryoprotective efficacy of lecithin and a mixture of cholesterol and lecithin was determined as the survivability of spermatozoa during cryopreservation. The viability of the reproductive cells was assessed by the mobility immediately after thawing and after 5 hours of incubation at $38{ }^{\circ} \mathrm{C}$. The mobility was determined on a SFA-500 sperm analyzer (OOO NPF BIOLA, Russia) as a proportion of spermatozoa with rectilinear motion at a velocity of more than $25 \mu \mathrm{m} / \mathrm{s}$. A standard lactose-glycerol yolk medium was the control.

Statistical processing was performed in the program Sig-maPlot 12.5 (Systat Software Inc., USA). Differences were considered statistically significant at $\mathrm{P}<0.05$. The experimental data (except the particle size dispersion) are presented as mean $(\bar{X})$ with a standard error $( \pm S E)$. For the sizes of particles produced by ultrasonic treatment, a size dispersion (SD) is shown.

Results. Dimensional characteristics of a suspension of lecithin obtained by sonication. Sonication is widely used for the preparation of nanosized liposomes. Unlike liposomes obtained by extrusion (punching a suspension through a polycarbonate membrane with pores of a certain size), in sonication the particle size essentially depends on the lipid composition and the properties of the lipid bilayer (Table 1).

1. Average size of phospholipid and phospholipidcholesterol particles after sonication $(n=4)$

\begin{tabular}{l|c|c}
\hline $\begin{array}{l}\text { Cholesterol } \\
\text { content, } \%\end{array}$ & $\begin{array}{l}\text { Average size of par- } \\
\text { ticles, } \mathrm{nm}(\bar{X} \pm \mathrm{SE})\end{array}$ & $\begin{array}{l}\text { Dispertion of particles (SD) } \\
\text { within the sample, nm }\end{array}$ \\
\hline 0 & $41 \pm 7$ & 51 \\
8 & $43 \pm 13$ & 42 \\
18 & $66 \pm 6$ & 56 \\
33 & $62 \pm 11$ & 46 \\
50 & $3476 \pm 532$ & 2978 \\
\hline N o t e. In determining the average size, the difference between the \\
mean values for particles in different samples is considered.
\end{tabular}

After sonication, the size of the liposomes formed in the suspension, depended on the lecithin/cholesterol ratio. Figure 1, A shows a typical histogram of the particle distribution in the sonicated LeciPRO 90 suspension. Ultrasonic treatment resulted in a polydisperse suspension with a two-, less often a three-modal distribution. There was a cluster with average liposome sizes of about $30 \mathrm{~nm}$ and a cluster of larger particles (about $100 \mathrm{~nm}$ ). In some samples, up to $1.5 \%$ liposomes were 300 to $1000 \mathrm{~nm}$ in diameter.

The increase in the duration of ultrasound treatment (from 5 to $20 \mathrm{~min}$ ) did not significantly affect the average size of the liposomes and their size distribution. LeciPRO 90 is a complex mixture of soybean polar lipids and other fatsoluble compaunds characteristic for plant raw materials, for example, carotenes. The most common components of commercial soybean lecithins of a similar class are phosphotidylcholine, phosphotidylethanolamine and phosphotidylinositol [20]. Liposomes resulted from such complex mixtures after sonication quite often demonstrate a complex polydisperse distribution in size [21], which corresponds to the results obtained by us. 
A
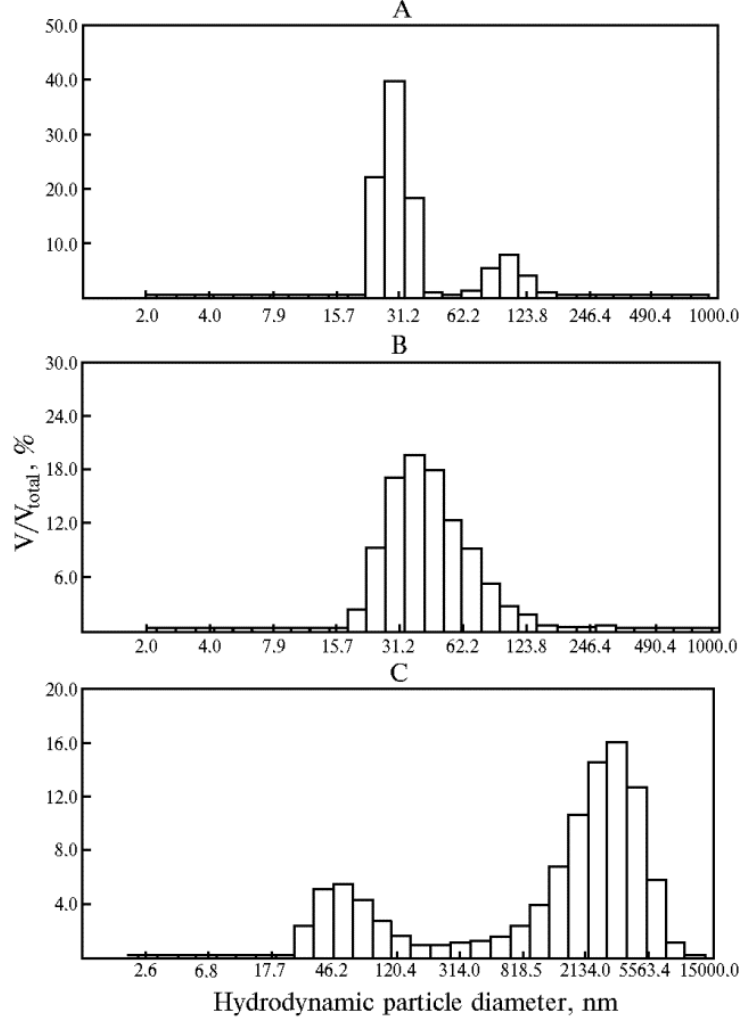

Fig. 1. Histogram of the size distribution of lipid particles formed from a mixture of cholesterol and lecithin LeciPRO 90 after sonication: A - without cholesterol, B $18 \%$ cholesterol, C $-50 \%$ cholesterol; $\mathrm{V} / \mathrm{V}_{\text {total }}$ - the ratio of the total volume of particles of the indicated size to the total volume of particles in the suspension. Ultrasonic disintegrator UZDN-2T (NPP Akadempribor, Russia, $22 \mathrm{kHz}$, $\left.60 \mathrm{~W} / \mathrm{cm}^{2}, 5 \mathrm{~min}\right)$.

The introduction of liposomes of LeciPRO 90 and low cholesterol $(8 \%)$ did not significantly affect the dimensional characteristics of the suspension. The average particle diameter did not change (see Table 1), and the type of their size distribution was similar to that observed for pure LeciPRO 90 (data not shown).

The increase in the cholesterol fraction up to 18 and $33 \%$ led to an increase in the average particle size and changed their distribution. After sonication, the mixtures formed a monodisperse suspension with an average parti-

cle size of $66 \pm 6$ and $62 \pm 11 \mathrm{~nm}$, respectively (see Table 1, Fig. 1, C).

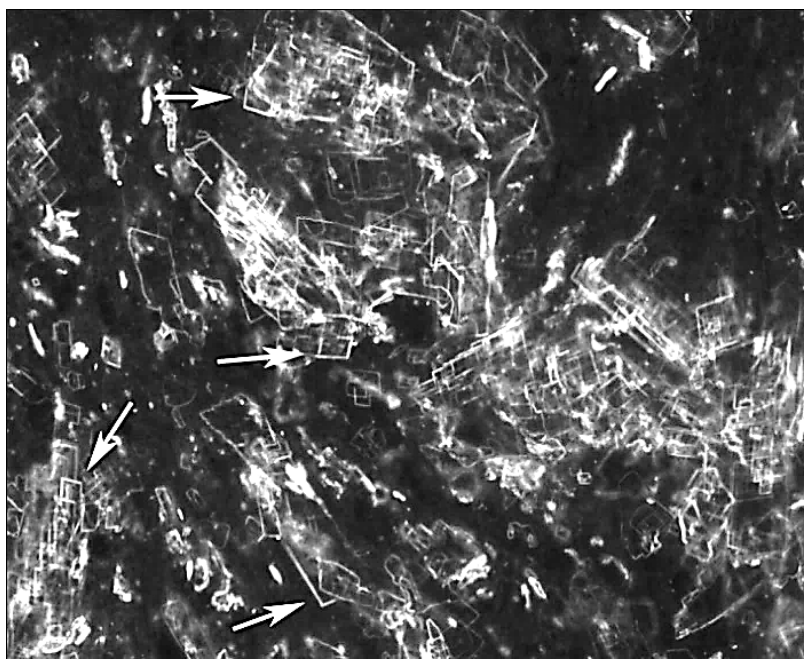

Fig. 2. Suspension obtained from a mixture of cholesterol and LeciPRO $90(50: 50 \mathrm{w} / \mathrm{w})$ after sonication. The arrows indicate particles with a shape characteristic of cholesterol crystals. Microscope Axio Scope FL (Carl Zeiss, Germany), ×400 magnification, dark field contrasting.

The $50 \%$ cholesterol corresponds to approximately 66 mole\%, the limiting amount of cholesterol that can retain the lipid bilayer formed from phosphotidylcholine. A higher cholesterol/lecithin ratio results in high cholesterol phospholipid lamellas and cholesterol

monohydrate crystals [22, 23]. The amount of cholesterol retained by the bilayer depends on the composition of the phospholipids [22]. For LeciPRO 90, this amount is unknown. From the data presented (see Table 1, Figure 1, C) it follows that the suspension of LeciPRO 90 and $50 \%$ cholesterol $(\approx 66$ mole\%) was fundamentally different from the others. The particle size in this mixture was more than an order of magnitude higher than that in suspensions from mixtures with a lower cholesterol concentration. In the suspension, two fractions of parti- 
cles (of 40-120 and 2500-3500 nm) were observed. Among the particles that were larger and more clearly distinguishable under a microscope, those with a shape characteristic of cholesterol crystals were seen (Fig. 2). At that, the suspension was sufficiently stable. During 24 hours, no signs of stratification were observed, in contrast to a suspension of pure cholesterol crystals which precipitated fairly quickly. It can be assumed that the large fraction is formed by complex structures of cholesterol crystals and cholesterol-phospholipid lamellae, and the small fraction is a liposome from a mixture with the maximum possible cholesterol content in the experiment.

Cryoprotection effect of liposomes formed from LeciPRO 90 and cholesterol. Comparison of spermatosoa viability in the control (standard sucrose-glycero-yolk medium) and when adding liposomes or lipid particles from a mixture of soybean lecithin and cholesterol in different ratios did not reveal a positive effect of cholesterol. Moreover, the viability of bull spermatozoa cryopreserved in the presence of mixtures with high cholesterol concentrations (18, 33 and $50 \%$ ) was lower than when frozen with LeciPRO 90 without cholesterol or when adding egg yolk (Fig. 3). Interestingly, the viability of spermatozoa gradually decreased as the cholesterol concentration increased up to $33 \%(\approx 50$ mole $\%)$. It is in this range of concentrations that cholesterol positively affects lipid chains, making the lipid bilayer stable and reducing fluctuations in the mobility of molecules.

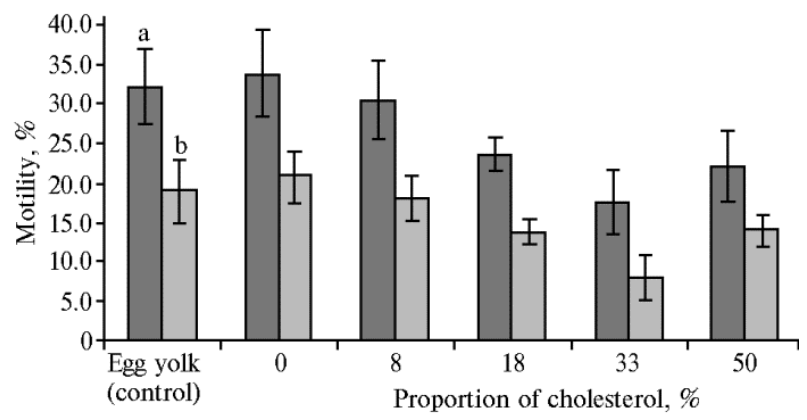

Fig. 3. Motility of bull spermatozoa frozen in the presence of mixtures of soybean lecithin and cholesterol 10 minutes (a) and 5 hours (b) after thawing $(\bar{X} \pm \mathrm{SE}, n=10)$.

Further increase in cholesterol concentration to $50 \%$ ( $\approx 66$ mole $\%)$ was accompanied by a certain increase in the viability of spermatozoa relative to the minimum value with a cho-

lesterol content of $33 \%$. Thus, a suspension of a lipid mixture with $50 \%$ cholesterol provided significantly $(\mathrm{P}<0.05)$ higher motility of spermatozoa 5 hours after thawing than a mixture with $33 \%$ cholesterol (see Fig. 3). It is in this range of cholesterol concentrations that the structure and size of lipid particles in the suspension change radically (see Table 1, Fig. 1, 2).

From this it can be concluded that the addition of cholesterol impairs the interaction of the lipids of the suspension with the cytoplasmic membrane of the spermatozoon. A direct negative effect of cholesterol on spermatozoa should manifest itself in a monotonous decrease of cryoprotection across the whole range of concentrations, which does not correspond to our data. This contradiction may be removed by the explanation that cryoprotective efficiency changes due to weakening the interaction between the lipid particles and the cells.

An increase in the proportion of cholesterol in membranes often leads to a decrease in the fusogenicity of liposomes [16, 17, 24]. However, this trend is observed in the range from 0 to 50 mole\% of cholesterol. With an increase in cholesterol content of more than 58 mole\%, the interaction of cholesterol and lipid molecules becomes energetically less favorable [25], which can increase the fusogenic property of liposomes compared to a minimum at 50 mole\%. Because of a complete rearrangement of the suspension structure which we observed in 
our experiments (see Fig. 1, 2), we can expect a significant change in the interaction between the lipids of the suspension and cell membranes of spermatozoa.

Thus, cholesterol added at $18-33 \%$ concentration to commercial soybean lecithin LeciPRO 90 increases the size particles after sonication of the mixture. At $50 \%$ concentration cholesterol completely changes the lipid suspension leading to formation of complex structures of phospholipid-cholesterol lamellae, cholesterol monohydrate crystals and liposomes. At $8 \%$ concentration cholesterol does not increase the cryoprotective effectiveness of lecithin, and at 18$50 \%$ concentrations the cryoprotection decreases. The most probably, this is due to deterioration in the interaction of lipids from the suspension with the cytoplasmic membrane of the spermatozoa.

\section{R E F E R E N C ES}

1. Layek S.S., Mohanty T.K., Kumaresan A., Parks J.E. Cryopreservation of bull semen: Evolution from egg yolk based to soybean based extenders. Anim. Reprod. Sci., 2016, 172: 1-9 (doi: 10.1016/j.anireprosci.2016.04.013).

2. Foulkes J.A. The separation of lipoproteins from egg yolk and their effect on the motility and integrity of bovine spermatozoa. J. Reprod. Fert. 1977, 49: 277-284 (doi: 10.1530/jrf.0.0490277).

3. Milova nov V.K., S e m e nova V.A. Doklady VASKHNIL, 1985, 11: 25-26 (in Russ.).

4. Erokhin A.S., D obrovol'ski i G.I. Molochnoe i myasnoe skotovodstvo, 2009, 5: 16-17 (in Russ.).

5. Shishova N.V., Ko mbarova N.A., D avydova G.A., S e raya O.Yu., Mirono va E.A., Abilov A.I., Pashovkin T.N., Gakhova E.N. Biologicheskie membrany, 2017, 34(3): 223-230 (in Russ.).

6. D a ri n-B e n n e t t A., Wh i t e I.G. Influence of the cholesterol content of mammalian spermatozoa on susceptibility to cold-shock. Cryobiology, 1977, 14(4): 466-470 (doi: 10.1016/0011-2240(77)90008-6).

7. $\quad \mathrm{N}$ a u k V.A. Struktura $\mathrm{i}$ funktsii spermiev sel'skokhozyaistvennykh zhivotnykh pri kriokonservatsii [Structure and function of farm animal cryopreserved semen]. Kishinev, 1991 (in Russ.).

8. Parks J.E., Ly n ch D.V. Lipid composition and thermotropic phase behavior of boar, bull, stallion, and rooster sperm membranes. Cryobiology, 1992, 29(2): 255-266 (doi: 10.1016/00112240(92)90024-V).

9. B lesbois E., Grasseau I., S e igneurin F. Membrane fluidity and the ability of domestic bird spermatozoa to survive cryopreservation. Reproduction, 2005, 129(3): 371-378 (doi: 10.1530/rep.1.00454).

10. D ro k i n S.I. Phospholipid distribution and fatty acid composition of phosphatidylcholine and phosphatidylethanolamine in sperm of some freshwater and marine species of fish. Aquatic Living Resources, 1993, 6: 49-56 (doi: 10.1051/alr:1993005).

11. Zidovetzki R., Levitan I. Use of cyclodextrins to manipulate plasma membrane cholesterol content: evidence, misconceptions and control strategies. Biochim. Biophys. Acta, 2007, 1768(6): 1311-1324 (doi: 10.1016/j.bbamem.2007.03.026).

12. Mocé E., Tomás C., Blanch E., Graham J.K. Effect of cholesterol-loaded cyclodextrins on bull and goat sperm processed with fast or slow cryopreservation protocols. Animal, 2014, 8(5): 771-776 (doi: 10.1017/S1751731114000226).

13. Rajoriya J.S., Prasad J.K., Ramteke S.S., Perumal P., Ghosh S.K., Singh M., Pande M., Srivastava N. Enriching membrane cholesterol improves stability and cryosurvival of buffalo spermatozoa. Anim. Reprod. Sci., 2016, 164: 72-81 (doi: 10.1016/j.anireprosci.2015.11.014).

14. M o c é E., B la n ch E., To má s C., G r a ha m J.K. Use of cholesterol in sperm cryopreservation: present moment and perspectives to future. Reprod. Domest. Anim., 2010, 45(Suppl 2): 57-66 (doi: 10.1111/j.1439-0531.2010.01635.x).

15. Fay y z M.H., A h mad M., A h mad N. Survival of buffalo bull spermatozoa: effect on structure and function due to alpha-lipoic acid and cholesterol-loaded cyclodextrin. Andrologia, 2016 (doi: 10.1111/and.12652).

16. Yang S.T., Kreutzberger A.J., Lee J., Kiessling V., Tamm L.K. The role of cholesterol in membrane fusion. Chem. Phys. Lipids, 2016, 199: 136-143 (doi: 10.1111/j.14390531.2010.01635.x).

17. Wang Z., Ma Y., Khalil H., Wang R., Lu T., Zhao W., Zhang Y., Chen J., $\mathrm{C}$ h e $\mathrm{n}$ T. Fusion between fluid liposomes and intact bacteria: study of driving parameters and in vitro bactericidal efficacy. Int. J. Nanomed., 2016, 11: 4025-4036 (doi: 10.2147/IJN.S55807).

18. Falck E., Patra M., Karttunen M., Hyvonen M.T., Vattulainen I. Lessons 
of slicing membranes: Interplay of packing, free area, and lateral diffusion in phospholipid/cholesterol bilayers. Biophys. J., 2004, 87: 1076-1091. (doi: 10.1529/biophysj.104.041368).

19. Natsional'naya tekhnologiya zamorazhivaniya $i$ ispol'zovaniya spermy plemennykh bykovproizvoditelei /Pod redaktsiei N.M. Reshetnikovoi, A.M. Abilova [National technology of freezing and use of pedigree bull sires' semen. N.M. Reshetnikova, A.I. Abilov (eds.)]. Moscow, 2008 (in Russ.).

20. S c holfi eld C.R. Composition of soybean lecithin. J. Am. Oil Chem. Soc., 1981, 58: 889892 (doi: 10.1007/BF02659652).

21. Ov chinnik ov Yu.A. Bioorganicheskaya khimiya [Bioorganic chemistry]. Moscow, 1987 (in Russ.).

22. H u a ng J., B u bolt z J.T., Fe ig e n s o n G.W. Maximum solubility of cholesterol in phosphatidylcholine and phosphatidylethanolamine bilayers. Biochim. Biophys. Acta, 1999, 1417: 89100 (doi: 10.1016/S0005-2736(98)00260-0).

23. F e ig e n s o n G.W., B u b o $1 \mathrm{t} \mathrm{z}$ J.T. Ternary phase diagram of dipalmitoyl-PC/dilauroylPC/cholesterol: nanoscopic domain formation driven by cholesterol. Biophys. J., 2001, 80: 2775-2788 (doi: 10.1016/S0006-3495(01)76245-5).

24. Ma Y., Wang Z., Zhao W., Lu T., Wang R., Me i Q., Che n T. Enhanced bactericidal potency of nanoliposomes by modification of the fusion activity between liposomes and bacterium. Int. J. Nanomed., 2013, 8(1): 2351-2360 (doi: 10.2147/IJN.S42617).

25. Huang J., Feigenson G.W. A microscopic interaction model of maximum solubility of cholesterol in lipid bilayers. Biophys. J., 1999, 76: 2142-2157 (doi: 10.1016/S0006-3495(99)77369-8). 